University of Windsor

Scholarship at UWindsor

1977

\title{
Influence of fringing fields on the field-induced quantum beats of atomic hydrogen
}

Gordon W. F. Drake

University of Windsor

Follow this and additional works at: https://scholar.uwindsor.ca/physicspub

Part of the Physics Commons

\section{Recommended Citation}

Drake, Gordon W. F.. (1977). Influence of fringing fields on the field-induced quantum beats of atomic hydrogen. Journal of Physics B: Atomic and Molecular Physics, 10 (5), 775-782.

https://scholar.uwindsor.ca/physicspub/40

This Article is brought to you for free and open access by the Department of Physics at Scholarship at UWindsor. It has been accepted for inclusion in Physics Publications by an authorized administrator of Scholarship at UWindsor. For more information, please contact scholarship@uwindsor.ca. 
Influence of fringing fields on the field-induced quantum beats of atomic hydrogen

This article has been downloaded from IOPscience. Please scroll down to see the full text article. 1977 J. Phys. B: At. Mol. Phys. 10775

(http://iopscience.iop.org/0022-3700/10/5/013)

View the table of contents for this issue, or go to the journal homepage for more

Download details:

IP Address: 137.207.184.30

The article was downloaded on 26/04/2013 at $15: 42$

Please note that terms and conditions apply. 


\title{
Influence of fringing fields on the field-induced quantum beats of atomic hydrogen $\dagger$
}

\author{
G W F Drake \\ Department of Physics, University of Windsor, Windsor, Ontario, Canada N9B 3P4
}

Received 26 October 1976

\begin{abstract}
Calculations are presented which include exactly the effects of fringing fields on the electric-field-induced quantum beats of atomic hydrogen. The results explain some recently observed phaseshifts of the quantum beats relative to the predictions of the 'sudden approximation'. The general features produced by finite rise-time and overshoot are discussed.
\end{abstract}

\section{Introduction}

The existence of quantum beats in atomic systems, particularly following beam-foil excitation (Sellin et al 1969, 1973, Alguard and Drake 1973, Andrä et al 1973, Gaupp et al 1974), is now well established and explanations have been developed for many of the qualitative features. (For a comprehensive review, see Andrä 1974.) A common assumption in most theoretical calculations of field-induced quantum-beat phenomena is that the perturbing field is switched on suddenly, say at time $t=0$ (see e.g. Alguard and Drake 1973). The quantum beats can then be viewed as resulting from interference amongst the coherent superposition of field-perturbed states such that the pure unperturbed initial state is recovered at $t=0$. As one exception, Wooten and Macek (1972) have given a partial treatment of switching-on effects in the quantum beats of atomic hydrogen. Pinnington et al (1973) discuss the observed suppression of beam-foil quantum beats by fringing fields of various geometries.

The purpose of this paper is to discuss the quantum beats recently reported by van Wijngaarden et al (1976). In their experiment, a transverse electric field was applied to a beam of hydrogen atoms initially prepared in the metastable $2 \mathrm{~s}$ state, and beats were observed in the induced $L y-\alpha$ radiation emitted in the direction parallel to the field. The field was switched on over a small but finite distance, and it was suggested tentatively that the fringing field may account for a pronounced phaseshift observed in the beats relative to calculations performed in the sudden approximation (see figure 1). Quantum-beat calculations, which include the theoretically calculated fringing field, are reported with the aim of explaining the observed 


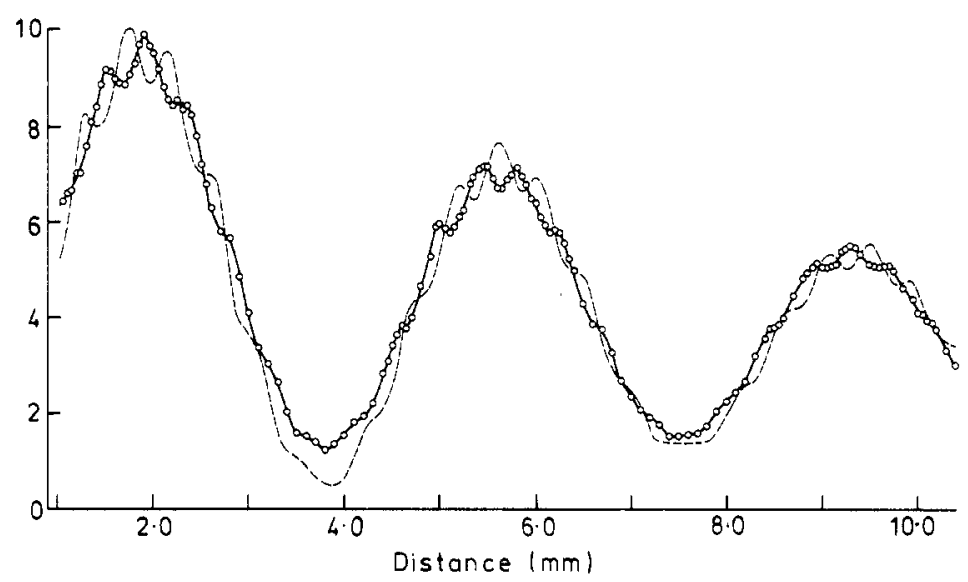

Figure 1. Comparison of the experimental quantum beats (circles) with the predictions of the sudden approximation (broken curve). The quenching field is $166.9 \mathrm{~V} \mathrm{~cm}^{-1}$ and the beam velocity is $4.79 \times 10^{8} \mathrm{~cm} \mathrm{~s}^{-1}$.

phaseshift and elucidating some of the general features produced by finite rise-time and overshoot. A very efficient method of integrating the time-dependent Schrödinger equation for problems of this type is used.

\section{Theory and computational method}

\subsection{Solution of the time-dependent Schrödinger equation}

An atomic hydrogen beam is assumed to be initially prepared in the $2 \mathrm{~s}$ metastable state. For purposes of discussing the quantum beats induced by electric fields up to about $10^{4} \mathrm{~V} \mathrm{~cm}^{-1}$, it is sufficient to consider only the manifold of 16 states with principal quantum number $n=2$. These are the hyperfine states $2 \mathrm{~s}_{1 / 2}(F=0,1)$, $2 \mathrm{p}_{1 / 2}(F=0,1)$ and $2 \mathrm{p}_{3 / 2}(F=1,2)$, each of which is $(2 F+1)$-fold degenerate. In this finite basis set, the time-dependent Schrödinger equation to be solved is (Bethe and Salpeter 1957)

$$
\begin{aligned}
& \mathrm{i} \frac{\mathrm{d} \boldsymbol{a}}{\mathrm{d} t}=\boldsymbol{U}(t) \boldsymbol{a} \\
& \boldsymbol{U}(t)=\boldsymbol{E}+F(t) \boldsymbol{V}
\end{aligned}
$$

in atomic units, where $a$ is the 16-component column vector of time-dependent state amplitudes, $E$ is the diagonal matrix of field-free eigenvalues, $V$ is the interaction matrix with the external electric field and $F(t)$ is the time-dependent field strength such that $F(t)=0$ for $t \leqslant 0$ and $F(t) \rightarrow F_{\infty}$ as $t \rightarrow \infty$. The field-free level widths $\Gamma_{i}$ are included in $E$ by the phenomenological relation

$$
E=E_{0}-\frac{1}{2} \mathrm{i} \Gamma \text {. }
$$

The components $a_{i}(t)$ of $\boldsymbol{a}$ are rapidly oscillating in time. Since equation (1) must be solved by numerical integration, it is computationally advantageous to transform to a new basis set with amplitudes $c(t)$ defined by the relation

$$
\boldsymbol{c}(t)=\exp \left(\mathbf{i} A^{-1} U_{\infty} \boldsymbol{A} t\right) \boldsymbol{A}^{-1} \boldsymbol{a}(t)
$$




$$
\boldsymbol{U}_{\infty}=\boldsymbol{E}+F_{\infty} \boldsymbol{V} \text {. }
$$

Here $\boldsymbol{A}$ is defined to be the matrix such that $\boldsymbol{A}^{-1} \boldsymbol{U}_{\infty} \boldsymbol{A}$ is diagonal. Note that $\boldsymbol{A}$ is not a unitary matrix since $U_{\infty}$ is non-Hermitian due to the inclusion of the imaginary level widths in the diagonal elements. It is readily seen that if $U_{\infty}$ is symmetric, then $\boldsymbol{A}^{-1}=\boldsymbol{A}^{\mathrm{T}}$ rather than $\boldsymbol{A}^{\dagger}$. The matrix $\boldsymbol{A}$ can be found by a simple generalization of Jacobi's method for matrix diagonalization. The state vector $c(t)$ then satisfies

$$
\mathrm{i} \frac{\mathrm{d} c}{\mathrm{~d} t}=f(t) \exp \left(\mathrm{i} A^{-1} U_{\infty} A t\right) A^{-1} \boldsymbol{V} A \exp \left(-\mathrm{i} A^{-1} U_{\infty} A t\right) \boldsymbol{c}(t)
$$

with

$$
f(t)=F(t)-F_{\infty}
$$

and

$$
c(0)=A^{-1} a(0) \text {. }
$$

Except that the transformation defined by $A$ is non-unitary, the above is equivalent to the interaction representation in the basis set of field-perturbed states. The advantages gained in this calculation are (i) $c(t)$ varies slowly with time, and (ii) $c(t)$ approaches a limiting value $c(\infty)$ as $F(t) \rightarrow F_{\infty}$. The numerical integration of (6) can therefore be stopped when $F(t)$ is sufficiently close to $F_{\infty}$.

The object of the above analysis is to describe the evolution of the wavefunction in time, assuming that the atom is in one of the four $2 s_{1 / 2}(F=0,1)$ hyperfine states at $t=0$. If these states are labelled by $i=1, \ldots, 4$, then

$$
\psi_{i}(t)= \begin{cases}\psi_{i} \mathrm{e}^{-\mathrm{i} E_{i} t} & t \leqslant 0 \\ \sum_{k, j}^{16} \psi_{k} A_{k j} c_{j}^{(i)}(t) \mathrm{e}^{-\mathrm{i} \bar{E}_{j} t} & t>0\end{cases}
$$

where the $\bar{E}_{j}$ are the complex eigenvalues of $U_{\infty}$ and $c_{j}^{(i)}(t)$ is the solution to (6) corresponding to the initial condition

$$
a_{j}(0)=\delta_{j, i} \text {. }
$$

In the limiting case where the field strength is turned on as a step function at $t=0$, the $c_{j}^{(i)}(t)$ are given by

$$
c_{j}^{(i)}(t)=\sum_{k} a_{k}(0) A_{k j}
$$

independent of $t>0$. This is equivalent to the analytic result obtained previously by Green's function methods in the same limiting case (Drake and Grimley 1975).

\subsection{Emission of radiation}

The field-induced electric-dipole transitions to the ground-state manifold (labelled by $f=1, \ldots, 4)$ proceed via mixing between the $2 \mathrm{~s}$ and $2 \mathrm{p}$ states. The transition rate is proportional to the quantity

$$
I(t)=\sum_{i, \hat{f}, \hat{e}}\left|\left\langle\psi_{i}(t)|\hat{e} \cdot r| \psi_{f}\right\rangle\right|^{2}
$$

where $\hat{\boldsymbol{e}}$ is the unit polarization vector and the sum over $\hat{\boldsymbol{e}}$ denotes two orthogonal 
vectors perpendicular to the direction of observation. Using (9), the transition matrix element for $t>0$ is

$$
\left\langle\psi_{i}(t)|\hat{\boldsymbol{e}} \cdot \boldsymbol{r}| \psi_{f}\right\rangle=\sum_{j} \mathrm{e}^{-\mathrm{i} \bar{E}_{j} t} \sum_{k}\left\langle\psi_{k}|\hat{\boldsymbol{e}} \cdot \boldsymbol{r}| \psi_{f}\right\rangle A_{k j} c_{j}^{(i)}(t)
$$

\section{Calculations and results}

A schematic diagram of the quenching cell used by van Wijngaarden et al (1976) is shown in figure 2. The fringing field near the entrance hole is minimized in their experiment by coating the inside of the end plate with a thin conducting layer. Except for the perturbing effect of the small entrance hole, the potential distribution across the end plate is then the same as in the free space between the parallel quenching plates held at potentials $+V$ and $-V$.

Figure 3 is a cross section through the quenching cell in the same plane as figure 2 showing the boundary conditions near the entrance hole. The electric field components $E_{i,}$ and $E_{\perp}$ (parallel and perpendicular to the beam axis) were calculated as described in the appendix with the results shown in figure 3. Since the field components vary in strength across the beam diameter (which is $\frac{3}{4}$ of the hole diameter), the curves show the field strengths averaged across the beam. The two significant features are that (i) there is a pronounced overshoot in $E_{\perp}$ just inside the entrance hole, and (ii) there is a strong pulse in $E_{\mid}$at the entrance hole, followed by a rapid decay to zero. One can picture the atoms experiencing an electric field vector which rotates from horizontal to vertical in the plane of the diagram as the atoms pass through the hole.

For purposes of calculating the quantum-beat pattern, we take the vertical direction in figures 2 and 3 (i.e. the direction of observation) to be the $z$ axis and the beam axis to be the $x$ axis. The field components $E_{\mid l}$ and $E_{\perp}$ appear to be time dependent in the co-moving atomic frame due to the motion of the atoms along the beam

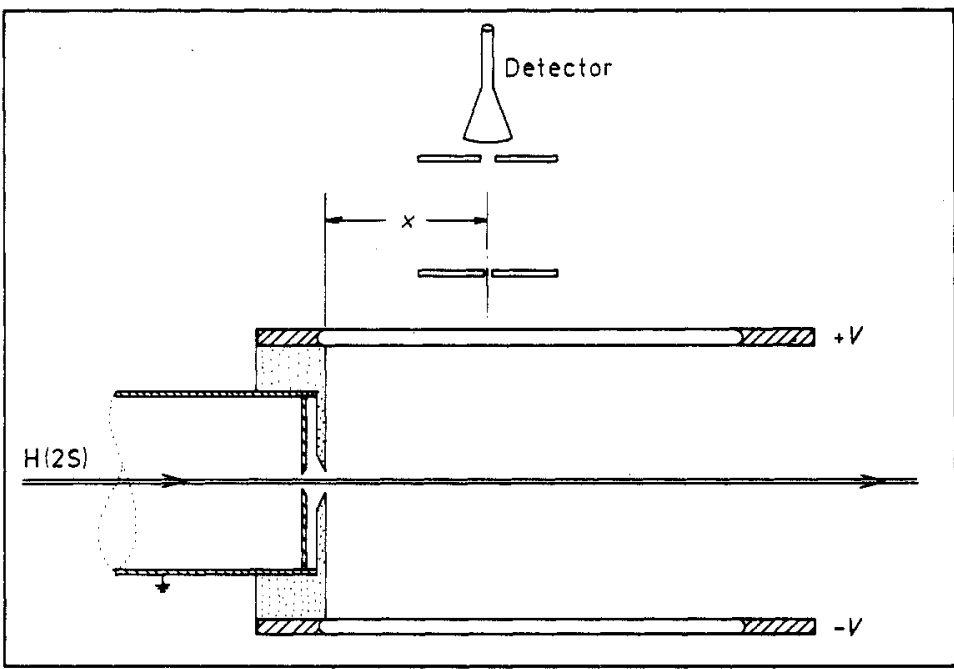

Figure 2. Schematic diagram of the apparatus. 


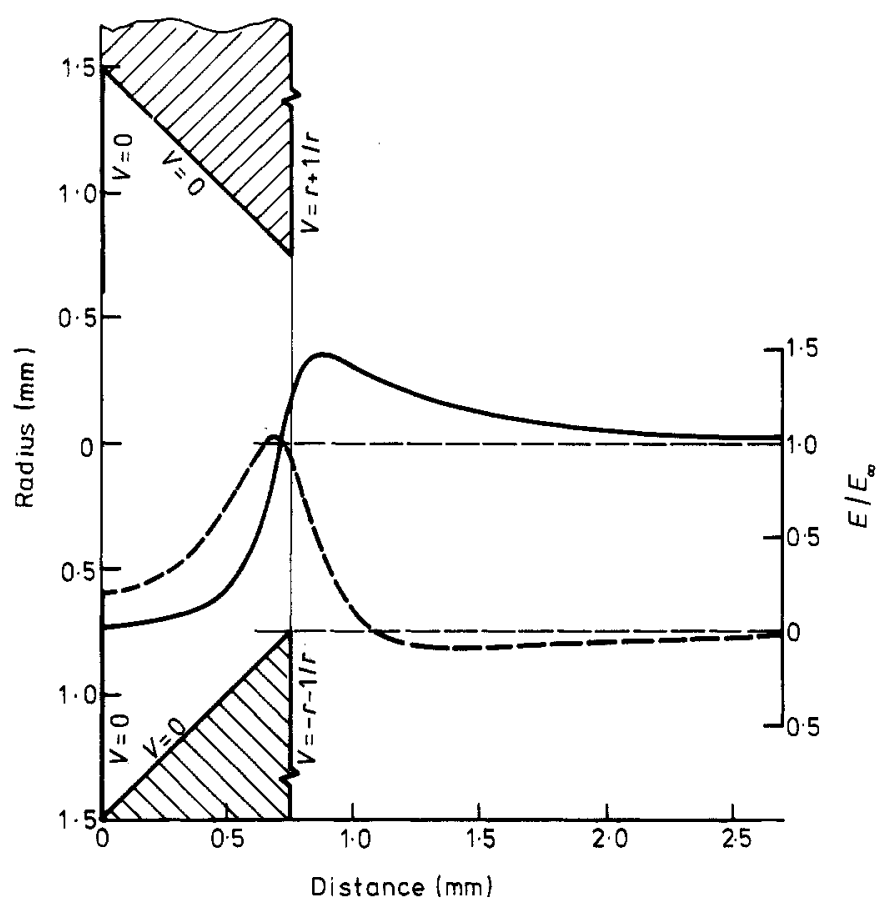

Figure 3. Cross section through the quenching cell showing the electric field boundary conditions near the entrance hole ( $r$ is measured in units of the hole radius) and the calculated field components $E_{\|}$and $E_{\perp}$ parallel and perpendicular to the (horizontal) beam axis. The full curve is $E_{\perp} / E_{x}$, and the broken curve is $E_{\|} / E_{x}$, where $E_{x}=166.9 \mathrm{~V} \mathrm{~cm}^{-1}$ is the asymptotic value of $E_{\perp}$.

axis. Since $E_{\|}$and $E_{\perp}$ have different time dependences (see figure 3) the term $f(t) V$ in equation (6) must be replaced by the sum of two terms

$$
f(t) \boldsymbol{V} \rightarrow f_{\perp}(t) \boldsymbol{V}_{\perp}+f_{\mid}(t) \boldsymbol{V}_{\mathrm{i}}
$$

where $V_{\perp}$ contains the matrix elements of $-e z$ and $V_{||}$contains the matrix elements of $-e x$ in the atomic frame of reference. The sum over polarization vectors in (12) in the above coordinate system contains the two contributions $\hat{e}_{x}$ and $\hat{\boldsymbol{e}}_{y}$. The calculation is simplified by summing instead over the equivalent spherical tensor components $e_{ \pm}=\mp\left(e_{x} \pm i e_{y}\right) / \sqrt{2}$ so that

$$
\sum_{\hat{e}}|\hat{e} \cdot r|^{2}=\sum_{ \pm}\left|\boldsymbol{r}_{ \pm}\right|^{2} .
$$

All the matrix elements between particular hyperfine states are conveniently expressed in terms of $3 j$ and $6 j$ symbols as described by Edmonds (1960). An explicit expression is given by Drake and Lin (1976).

To separate the effects produced by $E_{\perp}$ and $E_{i \mid}$, a calculation was first done with $E_{\mid}=0$ and the $E_{\downarrow}$ shown in figure 3 . In all the calculations, the asymptotic field strength far from the end plate is $166.9 \mathrm{~V} \mathrm{~cm}^{-1}$ and the beam velocity is $4.79 \times 10^{8} \mathrm{~cm} \mathrm{~s}^{-1}$ as determined by van Wijngaarden et al (1976). Each interval of length $1 \mathrm{~mm}$ along the beam axis therefore contains approximately two fine-structure oscillations. The resulting quantum-beat curve is compared in figure 4 (chain curve) with the 'sudden approximation' curve from figure 1. The arrow shows the point at 


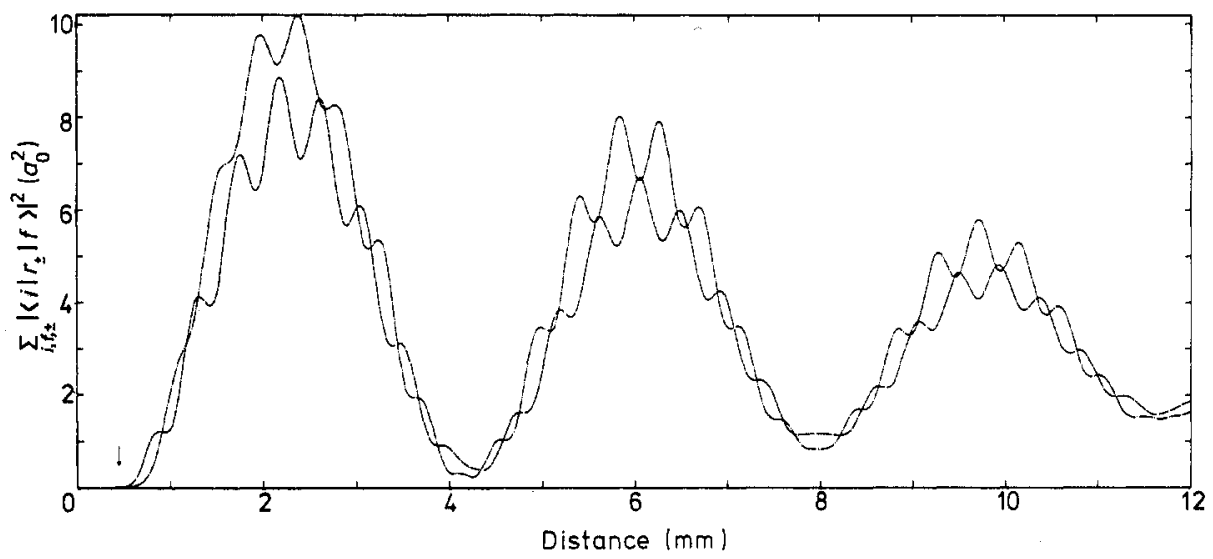

Figure 4. Comparison of sudden approximation (broken curve) with exact calculation including only $E_{\perp}$ (chain curve). The distance scale has the same origin as in figure 3 , and the arrow shows the point at which the field is switched on in the sudden approximation.

which the field is turned on in the sudden approximation adjusted so that the phases of the main Lamb shift oscillations approximately agree. The relative phase of the rapid fine-structure oscillations is clearly shifted by about $\pi$ so that the chain curve now agrees with the experimental curve in figure 1 . However, the chain curve still dips too low in the valleys, particularly at the first minimum near $4 \mathrm{~mm}$. This defect is corrected by including the $E_{\|}$field in the calculation as shown by the broken curve in figure 5. The shape near the peaks is nearly unchanged, but the intensity at the first minimum is increased by about a factor of three. The final theoretical curve, including an average over the geometrical slit function of the apparatus, is compared with the experimental curve in figure 6 . The overall agreement is now quite satisfactory. The small remaining discrepancies near the peaks can probably be accounted for by minor imperfections in the electric field calculation (such as averaging the field over the beam diameter) and small variations in the sensitivity of the experiment.

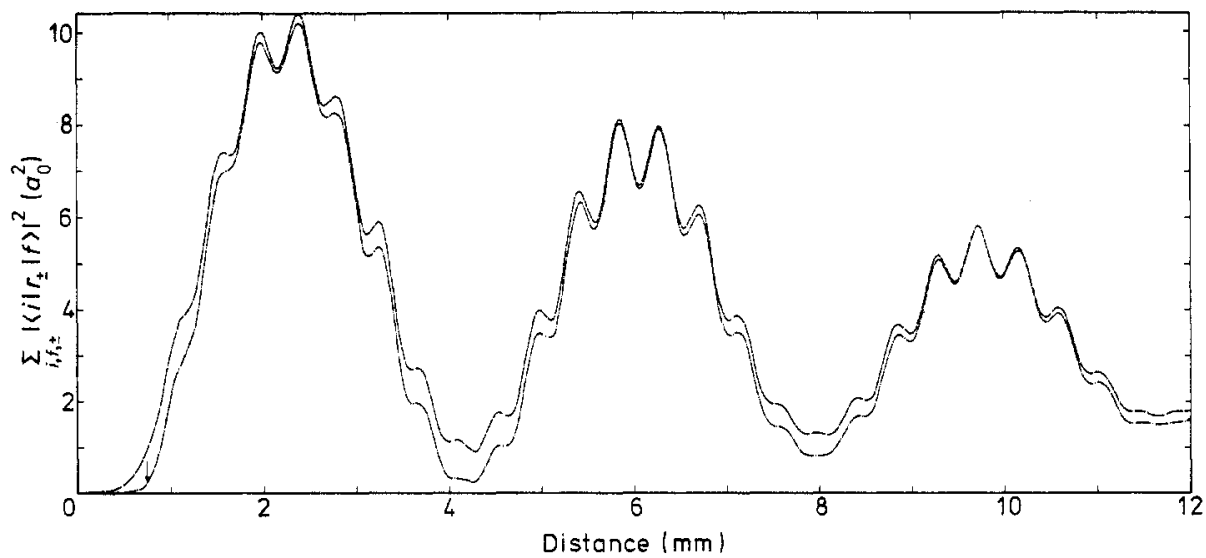

Figure 5. Comparison of exact calculation including only $E_{\perp}$ (chain curve) with the calculation including both $E_{\perp}$ and $E_{1:}$ (broken curve). The arrow shows the location of the entrance hole. 


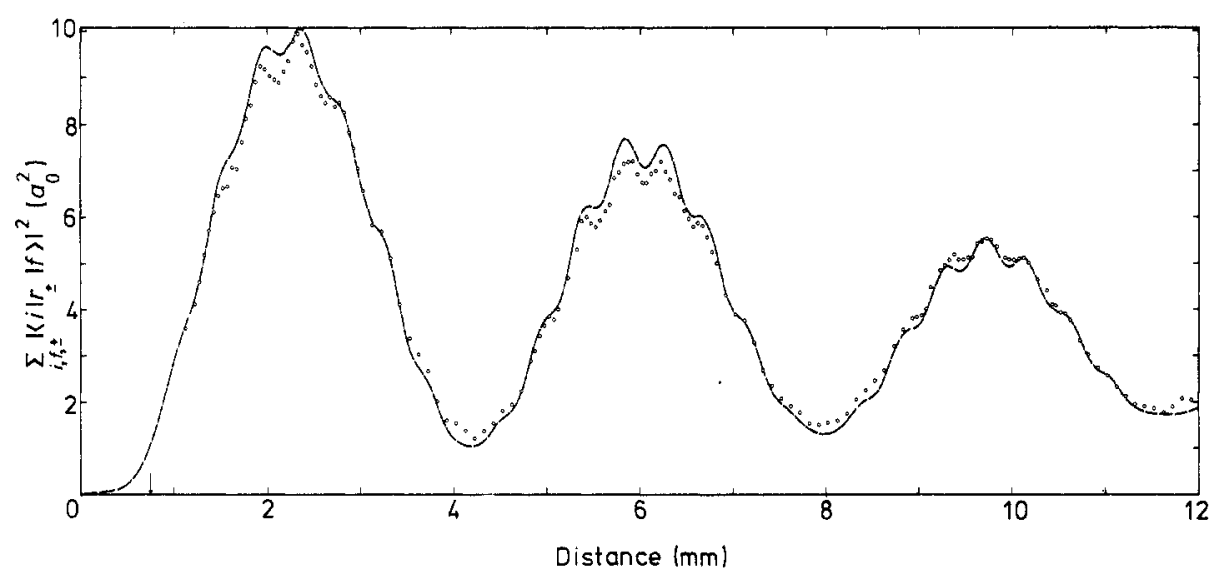

Figure 6. Comparison of final theoretical curve including instrumental broadening, with the experimental points (circles). The experimental points are multiplied by an arbitrary vertical scaling factor.

The only adjustable parameters are an overall vertical scaling factor and a horizontal shift of origin for the experimental points to bring them into the best possible agreement with theory. The horizontal shift of $0.15 \mathrm{~mm}$ is within the experimental margin of error for locating the absolute position of the entrance hole.

\section{Discussion}

The deviations in the experimental quantum-beat pattern from the sudden approximation are accounted for primarily by the overshoot in $E_{\perp}$ near the entrance hole. The presence of a finite rise-time in $E_{\perp}$ tends only to suppress the amplitude of the beats and cannot by itself account for the phaseshift. The overshoot has the opposite effect of increasing the amplitude while shifting the relative phases. The pulse in $E_{\|}$ near the entrance hole has very little effect on either the amplitudes or the phases near the peaks, but it substantially decreases the depth of the first few valleys.

An examination of the asymptotic state amplitudes $c_{j}^{(n)}(\infty)$ shows that the amplitudes for $2 \mathrm{p}_{1 / 2}$ and $2 \mathrm{p}_{3 / 2}$ are increased at the expense of $2 \mathrm{~s}_{1 / 2}$ relative to the suddenapproximation values. The same asymptotic results could be obtained in the sudden approximation by assuming that the atoms were initially in an appropriate coherent superposition of $2 \mathrm{~s}_{1 / 2}, 2 \mathrm{p}_{1 / 2}$ and $2 \mathrm{p}_{3 / 2}$ states instead of pure $2 \mathrm{~s}_{1 / 2}$. It is therefore necessary to examine field-switching effects carefully before using the quantum-beat pattern to deduce the initial state amplitudes. It is also necessary to correct for field-switching effects if one hopes to obtain accurate transition frequencies from the Fourier transform of the quantum-beat pattern, since the Fourier lineshapes change from Lorentzian form to resonance form as the phase is shifted (van Wijngaarden et al 1976). The general lineshape is a linear superposition of the two.

\section{Appendix. Electric field calculation}

For purposes of calculating the field near the entrance hole, we approximate the quenching cell by an infinite cylinder whose axis lies along the beam axis, together with the boundary condition

$$
V(r, \theta, z) \rightarrow V_{0} r \sin \theta \quad \text { as } \quad r \rightarrow \infty
$$


in cylindrical coordinates. Choosing the unit of distance to be the radius of the hole, the boundary condition on the conducting end plate is

$$
V_{\text {end }}(r, \theta)=V_{0}\left(r+r^{-1}\right) \sin \theta \quad r \geqslant 1
$$

so that $\partial V_{\text {end }} / \partial r=0$ at $r=1$. With these boundary conditions, the potential $\Phi(r, \theta, z)$ at an arbitrary point inside the cell can be written in the form

$$
\Phi(r, \theta, z)=\Phi_{1}(r, z) \sin \theta
$$

where $\Phi_{1}$ satisfies the Laplacian equation

$$
\left[\left(r \frac{\partial}{\partial r}\right)\left(r \frac{\partial}{\partial r}\right)+r^{2} \frac{\partial^{2}}{\partial z^{2}}-1\right] \Phi_{1}(r, z)=0 .
$$

This elliptic equation can be solved iteratively by dividing the $(z, r)$ plane into a grid of step size $h$ and approximating the derivatives by finite differences, as described for example by Sheldon (1960). If $z=i h$ and $r=j h$ at the grid point $(i, j)$, then the finite difference equation is

$\Phi_{i, j}\left(1+4 j^{2}\right)=j\left(j+\frac{1}{2}\right) \Phi_{i, j+1}+j\left(j-\frac{1}{2}\right) \Phi_{i, j-1}+j^{2}\left(\Phi_{i+1, j}+\Phi_{i-1, j}\right)$.

The semi-infinite plane $z \geqslant 0, \rho \geqslant 0$ can be truncated at values $z_{\max }$ and $r_{\max }$ equal to a few hole radii by using the approximate boundary condition along the line $r=r_{\max }$

$$
\Phi_{1}\left(r_{\max }, z\right)=V_{0}\left[r_{\max }+r_{\max }^{-1}\left(1-\frac{A z}{\left(r_{\max }^{2}+z^{2}\right)^{1 / 2}}\right)\right]
$$

with $A=\left(r_{\max }^{2}+z_{\max }^{2}\right)^{1 / 2} / z_{\max }$. The above corresponds to the exact solution if the boundary condition (A.2) extends to the origin. With these approximations, results accurate to one per cent or so can be obtained with a manageable grid size. The rate of convergence is greatly increased by use of the 'over relaxation' method discussed by Sheldon (1960).

\section{References}

Alguard M J and Drake C W 1973 Phys. Rev. A 8 27-36

Andrä H J 1974 Phys. Scr. $9257-80$

Andrä H J, Dobberstein P, Gaupp A and Wittmann W 1973 Nucl. Instrum. Meth. $110301-4$

Bethe H A and Salpeter E E 1957 Quantum Mechanics of One- and Two-Electron Atoms (Berlin: Springer-Verlag)

Drake G W F and Grimley R B 1975 Phys. Rev. A 11 1614-20

Drake G W F and Lin C.P 1976 Phys. Rev. A $141296-8$

Edmonds A R 1960 Angular Momentum in Quantum Mechanics (Princeton, NJ : Princeton University Press) Gaupp A, Andrä H J and Macek J 1974 Phys. Rev. Lett. 32 268-71

Pinnington E H, Berry H G, Desesquelles J and Subtil J L 1973 Nucl. Instrum. Meth. 110 315-20

Sellin I A, Moak C D, Griffin P M and Biggerstaff J A 1969 Phys. Rev. 188 217-21

Sellin I A, Mowat J R, Peterson R S, Griffin P M, Laubert R and Hazelton H H 1973 Phys. Rev. Lett. 31 1335-7

Sheldon J W 1960 Mathematical Methods for Digital Computers ed A Ralston and H W Wilf (New York: Wiley)

van Wijngaarden A, Goh E, Drake G W F and Farago P S 1976 J. Phys. B: Atom. Molec. Phys. 9 2017-25

Wooten J W and Macek J H 1972 Phys. Rev. A 5 137-40 